\title{
Comparative Evaluation of Four Toluidine Blue Stainings for Rat Femur Effect
}

\author{
Tian Gao ${ }^{1}$, Fuhou Chang ${ }^{1}$, Xiaohong $\mathrm{Wu}^{1}$, Yunfeng Xiao \\ ${ }^{1}$,Xiaozhen $\mathrm{Po}^{2 *}$ (corresponding author)
}

1 Center for safty evaluation of drugs,Inner Mongolia Medical University, Hohhot, $\mathrm{MO}, 010110$

2 Basic Medicine College,Inner Mongolia Medical University, Hohhot, MO,010110

\begin{abstract}
Objective to compare different ratio of toluidine blue dye solution on the advantages and disadvantages of rat femur dyeing. Methods: the rat femur of materials, fixation, embedding, and sectioning, respectively with different ratio of toluidine blue dye solution dyed, observation of femoral morphology change of the organization. Results show: borax toluidine blue dye solution is better than the other toluidine blue dye solution.
\end{abstract}

KEYWORDS: Rat; Femur; Toluidine Blue; Histomorphology

\section{Introduction}

Toluidine blue as a basic dye in the skin, lungs, trachea, brain and spinal cord special staining, has achieved good results, which is clear and background in contrasting colors. Morphological study of rats femur plays a very important role in evaluating the establishment fractures and osteoporosis model, and the number of bone cells toluidine blue staining method for displaying this new bone tissue calcium deposition into the statistics observed cartilage degeneration, necrosis calcified cartilage, fractured cartilage matrix, collagen fibers and other pathological changes. Found in the technical literature conventional toluidine blue staining is not a good coloring process in toluidine blue stained tissue sections of femur, clearly show tissue morphology. In this experiment, the femur for the study, comparative study of different processes of toluidine blue staining of the femur coloring effect, provide a technical basis for the quantitative study of the femoral morphology evaluation.

\section{Materials and Methods}




\section{A. Experimental Material.}

Inspection femur specimens. (Wistar rats were purchased from the Experimental Animal Center of Inner Mongolia University Certificate of Conformity: SCXK (Mongolia) 2005-0004] weighed anesthesia, euthanasia, from rat femur, 10\% neutral formalin fixed.)

\section{B. Main Instruments and Reagents}

Instrument Leica RM2135 Rotary Microtome, Leica EG 1150H tissue embedding machine, Leica DM2000 microscope with LAS4.1 image analysis system for image acquisition.

Reagents toluidine blue (import-packing), pH buffers - mixed phosphate (Tianjin Yong Sheng Fine Chemical Co., Ltd.), borax (Tianjin Yong Sheng Fine Chemical Co., Ltd.), pure acetone (Sinopharm).

\section{Dye Formulation}

$0.5 \%$ toluidine blue dye: toluidine blue $0.5 \mathrm{~g}$, distilled water $100 \mathrm{ml}$, stored at room temperature.

$1 \%$ Borax - toluidine blue dye: borax $0.5 \mathrm{~g}$, toluidine blue $0.5 \mathrm{~g}$, dissolved in $50 \mathrm{ml}$ of distilled water, until all dissolved filtration, stored at room temperature.

\section{Tissue Selection, Fixation, Embedding, Sectioning and Packet Processing}

Take femur, neutral formalin-fixed, by gradient alcohol dehydration, xylene, after dipping wax joint section close to the bottom of the embedding cassette. The embedded block is fixed to the dressing good slicer, slice thickness 5um continuous cut, the slices will be divided into four groups, each 10, were used four kinds of toluidine blue staining dye.

\section{E. Sections Were Deparaffinized and Stained}

The four of the slices in xylene (I) $10 \mathrm{~min}$, xylene (II) $5 \mathrm{~min}, 100 \%$ ethanol $5 \mathrm{~min}$, $100 \%$ ethanol $5 \mathrm{~min}$, $95 \%$ ethanol $5 \mathrm{~min}$, $95 \%$ ethanol $5 \mathrm{~min}, 80 \%$ aqueous ethanol to $5 \mathrm{~min}$.

$0.5 \%$ toluidine blue dye staining at room temperature $10 \mathrm{~min}$, washed with distilled water three times, each 20s.

$0.5 \%$ toluidine blue dye staining 10min (preheating 20min), washed with distilled water three times, each time 20s, phosphate buffer washed twice.

$0.5 \%$ toluidine blue dye staining $10 \mathrm{~min}$ (preheating $20 \mathrm{~min}$ ), washed with distilled water three times, each time 20s, 2 min separation in pure acetone, a little water.

$1 \%$ borax - toluidine blue dye dropped on sectioned and stained $3 \mathrm{~min}$, rinse the bottle sliced excess dye, drain water, 95\% ethanol rapid differentiation.

After four sections were stained upward gradient alcohol, xylene, neutral resin were mounted to microscopic examination.

\section{Result}

Respectively using conventional toluidine blue staining (Fig. 1), toluidine blue phosphate buffer staining (Fig. 2), toluidine blue - pure acetone staining (Fig. 3), 
borax - toluidine blue staining (Fig. 4 ), tissue sections of rat femur were stained, the results shown in Fig. The figure shows, respectively, by conventional toluidine blue staining, toluidine blue - stained phosphate buffer, toluidine blue - stained pure acetone femoral growth plate cartilage tissue calcification area and trabecular bone calcification dyed blue and purple, but the matrix and bone marrow cells have not yet colored. By Borax - toluidine blue dye staining the femoral growth plate cartilage calcification region area, trabecular bone calcification dyed blue and purple, lightly stained bone marrow cells, light blue background, contrasting.

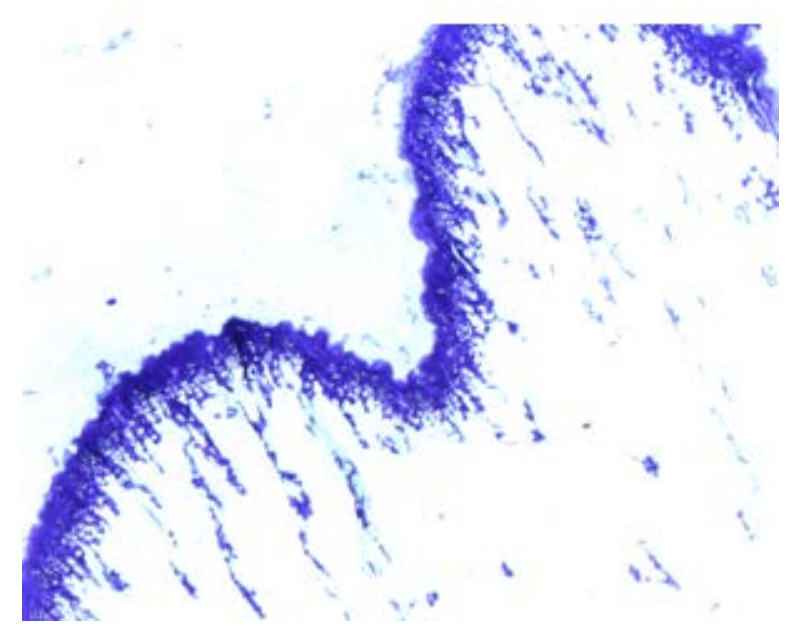

Fig. 1 Conventional toluidine blue staining

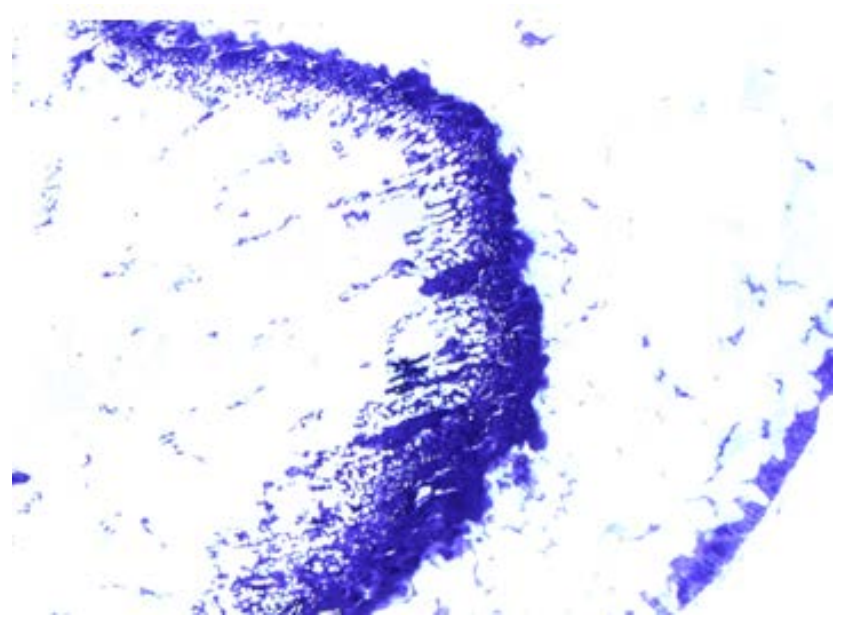

Fig. 2 Toluidine blue - stained phosphate buffer 


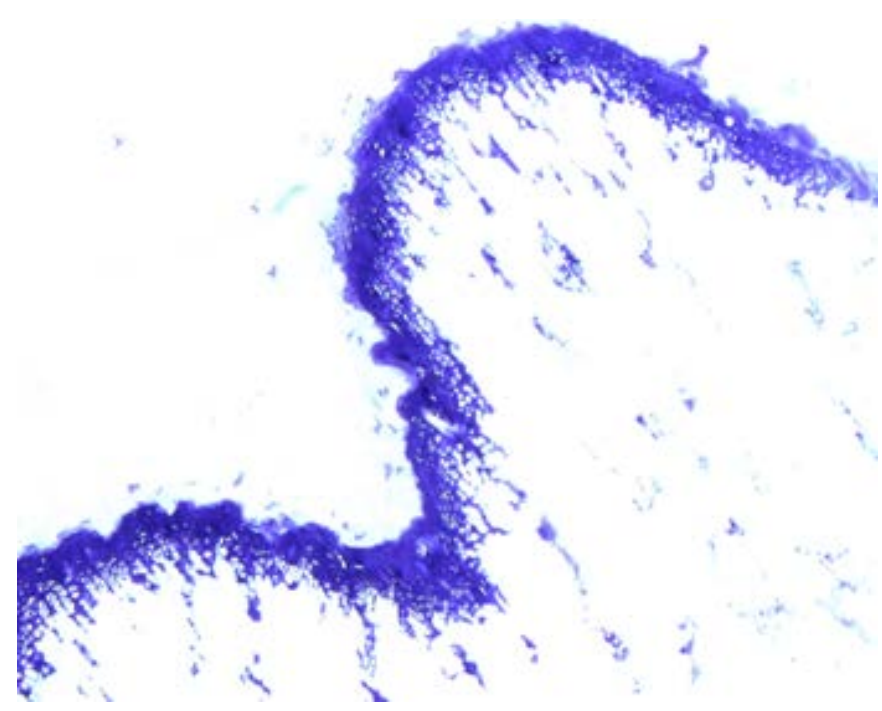

Fig. 3 Toluidine blue-stained pure acetone

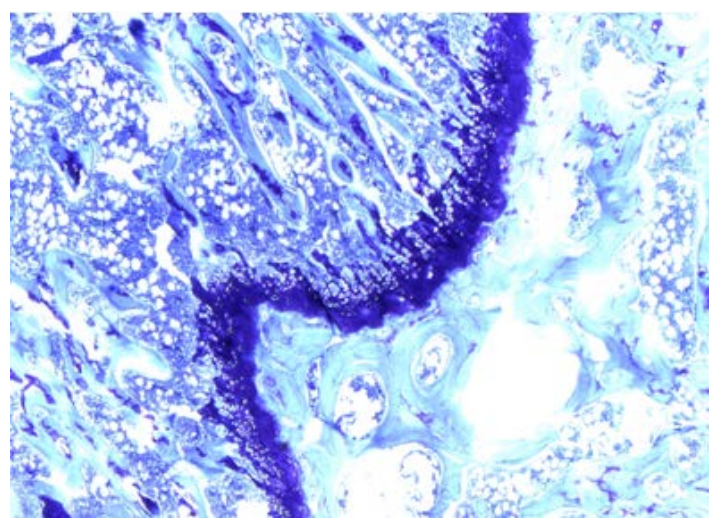

Fig.4 Borax - toluidine blue dye staining

\section{Result Discussion}

\section{A. The Basic Principle of Toluidine Blue Stained}

Toluidine blue is commonly used synthetic dyes, quinone imine dyes belong, these dyes generally contain both a chromophore, is an amine, a quinoid ring, a chromogen to form a color. Apart from the dye chromophore, but also contains the original color can make to the organization and other pollutants produced by affinity help chromophore, can promote dye produce ionized salts, chromophore help the organization produce dyeing ability, the upper sections tissue staining. It is alkaline toluidine blue dye, cationic dye has the role of the organization and the acidic substance which is a combination of a cationic dye, so that the nucleus can be dyed blue.

\section{B. Effect of Temperature on Toluidine Blue Stained}

Toluidine blue (Toluidine blue, TB) is an important biological stain and photosensitizer. Study on the thermal stability of the molecule in the TB $25-250{ }^{\circ} \mathrm{C}$ range using infrared spectroscopy. In the range of 25-160 ${ }^{\circ} \mathrm{C}$ TB infrared absorption spectrum did not change significantly, indicating its molecular structure remains stable in this temperature range. In the range $160--248{ }^{\circ} \mathrm{C}$, with increasing 
temperature TB decreased in 3687,3429,1832cm-1 and an absorption peak at $1756 \mathrm{~cm}-1$, especially when the temperature rises to $248{ }^{\circ} \mathrm{C}$, four absorption peak decreases rapidly, which may be caused by $\mathrm{N}-\mathrm{H}, \mathrm{C}-\mathrm{N}$ and $\mathrm{C}-\mathrm{S}$ bond due to rupture. The results show that TB has a wide temperature range, below $160{ }^{\circ} \mathrm{C}$ has good thermal stability, so the temperature will increase to some extent, solubility and coloring dyes. In this experiment, toluidine blue dye can be suitably heated, thereby ensuring the slice has good dyeing effect.

\section{Effect of PH on the Toluidine Blue Stained}

Since toluidine blue containing two chromophore and chromophore 2 aid for organizations with high affinity basophilic dye, studies show that when the $\mathrm{pH}$ value is between 4 to 9, will increase the clarity of the nucleolus, which will help the cell structure was observed. In the experiment with a phosphate buffer (pH7.2-7.4) weakly alkaline, while the strongly alkaline aqueous solution of borax (pH9.3), in its capacity as a buffer formulated with respect to toluidine blue phosphate buffer is greatly improved color strength tissue cells, tissue cells more clearly, to some extent, in greatly improving the efficiency of the observer.

\section{Effect of Dyeing Time of Toluidine Blue}

Staining time by the organization Chromatophilic substances affinity dyes and dye preparation time and reuse decisions. Experiment compared to the other three methods borax toluidine blue dye can quickly colored background with organizations such decomposition is obvious, but in order to prevent borax toluidine blue staining caused by long time over blue background and observe the effect, should Note that control staining time.

\section{E. Effect of Separation Time and Separation Agent on Toluidine Blue Stained}

Dichroic dye is critical, mainly for off excess dye which, in the toluidine blue staining, the use of 95\% ethanol separation, reduces background and organizational boundaries observed more pronounced in favor of observation. However, due to different slice thickness, should pay attention to color separation using dichroic agent is moderate for too long will lead to slice fade, stain and affect the next step. The experiment used 95\% ethanol rapidly dividing, both to ensure the boundaries between the background and the organization obviously not cause tissue discoloration and affect the next dyeing.

\section{F. Other Factors Affect the Toluidine Blue Dye}

During operation of the experiment will encounter other problems, such as xylene characteristic change toluidine blue staining reaction time is too long the transparent metachromatic reaction xylene may be reversed in the experiment will be transparent as much as possible in the time control 3 in addition, the phosphate buffer is easy to react with water, calcium, magnesium, place too long is also easy to produce precipitation, distilled water should be used during the experiment using now;; minutes due to alkaline toluidine blue dye, with a clear the easy dyeing, such easy dyeing when most are destroyed by alcohol dehydration, and therefore can not be sliced stained by alcohol dehydration, and washed with water as well. 


\section{REFERENCE:}

[1] Dai Xiaowei, Huang Lan, Xu Yanfeng, Ma Chunmei. Toluidine Blue Expand the Application Range in Tissue Staining, the Laboratory Animal and Comparative Medicine 2010 Vol 06 440-444 30 Pages, R361.2,10.3969 / J. Issn.1674-5817.2010.06.011.

[2] Zhao Xianyin, Li Xiaohong; Zhu Xiaoshu; Tong Yao. The Jaw and Femur Trabecular Bone Structure in Ovariectomized Rats, Xi'an Jiaotong University, Medicine Edition, CAS CSCD 2004 Vol. 25 No. 3 285- 287.

[3] Qinyan Li, Xu Sent Ning, Hu. Toluidine Blue Variable Temperature IR Spectroscopy, Spectroscopy Laboratory, 2010,27 (6), 2011.3.4 Liaoning Provincial Department of Education Project, Dr. Shenyang University of Technology Start-Up Projects.

[4] Liu Shuangfeng, Fan Yongxing, Yuan Xue, etc. Effect of Ph on Borax Toluidine Blue-Stained, and Clinical Laboratory Medicine, 2010,07 (11) R446. 